\title{
Specifying and Verifying Properties of Space*
}

\author{
Vincenzo Ciancia ${ }^{1}$, Diego Latella ${ }^{1}$, Michele Loreti $^{2}$, and Mieke Massink ${ }^{1}$ \\ 1 Istituto di Scienza e Tecnologie dell'Informazione 'A. Faedo', CNR, Italy \\ 2 Università di Firenze, Italy
}

\begin{abstract}
The interplay between process behaviour and spatial aspects of computation has become more and more relevant in Computer Science, especially in the field of collective adaptive systems, but also, more generally, when dealing with systems distributed in physical space. Traditional verification techniques are well suited to analyse the temporal evolution of programs; properties of space are typically not explicitly taken into account. We propose a methodology to verify properties depending upon physical space. We define an appropriate logic, stemming from the tradition of topological interpretations of modal logics, dating back to earlier logicians such as Tarski, where modalities describe neighbourhood. We lift the topological definitions to a more general setting, also encompassing discrete, graph-based structures. We further extend the framework with a spatial until operator, and define an efficient model checking procedure, implemented in a proof-of-concept tool.
\end{abstract}

\section{Introduction}

Much attention has been devoted in Computer Science to formal verification of process behaviour. Several techniques, such as run-time monitoring and modelchecking, are based on a formal understanding of system requirements through modal logics. Such logics typically have a temporal flavour, describing the flow of events along time, and are interpreted in various kinds of transition structures.

Recently, aspects of computation related to the distribution of systems in physical space have become more relevant. An example is provided by so called collective adaptive systems ${ }^{1}$, typically composed of a large number of interacting objects. Their global behaviour critically depends on interactions which are often local in nature. Locality immediately poses issues of spatial distribution of objects. Abstraction from spatial distribution may sometimes provide insights in the system behaviour, but this is not always the case. For example, consider a bike (or car) sharing system having several parking stations, and featuring twice as many parking slots as there are vehicles in the system. Ignoring the spatial dimension, on average, the probability to find completely full or empty parking stations at an arbitrary station is very low; however, this kind of analysis may

\footnotetext{
* Research partially funded by EU ASCENS (nr. 257414), EU QUANTICOL (nr. 600708), IT MIUR CINA and PAR FAS 2007-2013 Regione Toscana TRACE-IT.

${ }^{1}$ See e.g. the web site of the QUANTICOL project: http://www.quanticol.eu
}

J. Diaz et al.(Eds.): TCS 2014, LNCS 8705, pp. 222-235, 2014.

(C) IFIP International Federation for Information Processing 2014 
be misleading, as in practice some stations are much more popular than others, often depending on nearby points of interest. This leads to quite different probabilities to find stations completely full or empty, depending on the spatial properties of the examined location. In such situations, it is important to be able to predicate over spatial aspects, and eventually find methods to certify that a given formal model of space satisfies specific requirements in this respect. In Logics, there is quite an amount of literature focused on so called spatial logics, that is, a spatial interpretation of modal logics. Dating back to early logicians such as Tarski, modalities may be interpreted using the concept of neighbourhood in a topological space. The field of spatial logics is well developed in terms of descriptive languages and computability/complexity aspects. However, the frontier of current research does not yet address verification problems, and in particular, discrete models are still a relatively unexplored field.

In this paper, we extend the topological semantics of modal logics to closure spaces. As we shall discuss in the paper, this choice is motivated by the need to use non-idempotent closure operators. A closure space (also called $\breve{C} e c h$ closure space or preclosure space in the literature), is a generalisation of a standard topological space, where idempotence of closure is not required. By this, graphs and topological spaces are treated uniformly, letting the topological and graph-theoretical notions of neighbourhood coincide. We also provide a spatial interpretation of the until operator, which is fundamental in the classical temporal setting, arriving at the definition of a logic which is able to describe unbounded areas of space. Intuitively, the spatial until operator describes a situation in which it is not possible to "escape" an area of points satisfying a certain property, unless by passing through at least one point that satisfies another given formula. To formalise this intuition, we provide a characterising theorem that relates infinite paths in a closure space and until formulas. We introduce a model-checking procedure that is linear in the size of the considered space. A prototype implementation of a spatial model-checker has been made available; the tool is able to interpret spatial logics on digital images, providing graphical understanding of the meaning of formulas, and an immediate form of counterexample visualisation. ${ }^{2}$

Related work. We use the terminology spatial logics in the "topological" sense; the reader should be warned that in Computer Science literature, spatial logics typically describe situations in which modal operators are interpreted syntactically, against the structure of agents in a process calculus (see $[8,6]$ for some classical examples). The object of discussion in this research line are operators that quantify e.g., over the parallel sub-components of a system, or the hidden resources of an agent. Furthermore, logics for graphs have been studied in the context of databases and process calculi (see [7,15], and references), even though the relationship with physical space is often not made explicit, if considered at all. The influence of space on agents interaction is also considered in the literature on process calculi using named locations [11].

2 Due to lack of space all the proofs are omitted and can be found in [10]. 
Variants of spatial logics have also been proposed for the symbolic representation of the contents of images, and, combined with temporal logics, for sequences of images [12]. The approach is based on a discretisation of the space of the images in rectangular regions and the orthogonal projection of objects and regions onto Cartesian coordinate axes such that their possible intersections can be analysed from different perspectives. It involves two spatial until operators defined on such projections considering spatial shifts of regions along the positive, respectively negative, direction of the coordinate axes and it is very different from the topological spatial logic approach.

A successful attempt to bring topology and digital imaging together is represented by the field of digital topology $[22,25]$. In spite of its name, this area studies digital images using models inspired by topological spaces, but neither generalising nor specialising these structures. Rather recently, closure spaces have been proposed as an alternative foundation of digital imaging by various authors, especially Smyth and Webster [23] and Galton [17]; we continue that research line, enhancing it with a logical perspective. Kovalevsky [19] studied alternative axioms for topological spaces in order to recover well-behaved notions of neighbourhood. In the terminology of closure spaces, the outcome is that one may impose closure operators on top of a topology, that do not coincide with topological closure. The idea of interpreting the until operator in a topological space is briefly discussed in the work by Aiello and van Benthem [1,24]. We start from their definition, discuss its limitations, and provide a more fine-grained operator, which is interpreted in closure spaces, and has therefore also an interpretation in topological spaces. In the specific setting of complex and collective adaptive systems, techniques for efficient approximation have been developed in the form of mean-field / fluid-flow analysis (see [5] for a tutorial introduction). Recently (see e.g., [9]), the importance of spatial aspects has been recognised and studied in this context. In this work, we aim at paving the way for the inclusion of spatial logics, and their verification procedures, in the framework of mean-field and fluid-flow analysis of collective adaptive systems.

\section{Closure Spaces}

In this work, we use closure spaces to define basic concepts of space. Below, we recall several definitions, most of which are explained in [17].

Definition 1. A closure space is a pair $(X, \mathcal{C})$ where $X$ is a set, and the closure operator $\mathcal{C}: 2^{X} \rightarrow 2^{X}$ assigns to each subset of $X$ its closure, obeying to the following laws, for all $A, B \subseteq X$ :

1. $\mathcal{C}(\emptyset)=\emptyset$;

2. $A \subseteq \mathcal{C}(A)$;

3. $\mathcal{C}(A \cup B)=\mathcal{C}(A) \cup \mathcal{C}(B)$.

As a matter of notation, in the following, for $(X, \mathcal{C})$ a closure space, and $A \subseteq X$, we let $\bar{A}=X \backslash A$ be the complement of $A$ in $X$. 
Definition 2. Let $(X, \mathcal{C})$ be a closure space, for each $A \subseteq X$ :

1. the interior $\mathcal{I}(A)$ of $A$ is the set $\overline{\mathcal{C}(\bar{A})}$;

2. $A$ is a neighbourhood of $x \in X$ if and only if $x \in \mathcal{I}(A)$;

3. $A$ is closed if $A=\mathcal{C}(A)$ while it is open if $A=\mathcal{I}(A)$.

Lemma 1. Let $(X, \mathcal{C})$ be a closure space, the following properties hold:

1. $A \subseteq X$ is open if and only if $\bar{A}$ is closed;

2. closure and interior are monotone operators over the inclusion order, that is: $A \subseteq B \Longrightarrow \mathcal{C}(A) \subseteq \mathcal{C}(B)$ and $\mathcal{I}(A) \subseteq \mathcal{I}(B)$

3. Finite intersections and arbitrary unions of open sets are open.

Closure spaces are a generalisation of topological spaces. The axioms defining a closure space are also part of the definition of a Kuratowski closure space, which is one of the possible alternative definitions of a topological space. More precisely, a closure space is Kuratowski, therefore a topological space, whenever closure is idempotent, that is, $\mathcal{C}(\mathcal{C}(A))=\mathcal{C}(A)$. We omit the details for space reasons (see e.g., [17] for more information).

Next, we introduce the topological notion of boundary, which also applies to closure spaces, and two of its variants, namely the interior and closure boundary (the latter is sometimes called frontier).

Definition 3. In a closure space $(X, \mathcal{C})$, the boundary of $A \subseteq X$ is defined as $\mathcal{B}(A)=\mathcal{C}(A) \backslash \mathcal{I}(A)$. The interior boundary is $\mathcal{B}^{-}(A)=A \backslash \mathcal{I}(A)$, and the closure boundary is $\mathcal{B}^{+}(A)=\mathcal{C}(A) \backslash A$.

Proposition 1. The following equations hold in a closure space:

$$
\begin{aligned}
\mathcal{B}(A) & =\mathcal{B}^{+}(A) \cup \mathcal{B}^{-}(A) \\
\mathcal{B}^{+}(A) \cap \mathcal{B}^{-}(A) & =\emptyset \\
\mathcal{B}(A) & =\mathcal{B}(\bar{A}) \\
\mathcal{B}^{+}(A) & =\mathcal{B}^{-}(\bar{A}) \\
\mathcal{B}^{+}(A) & =\mathcal{B}(A) \cap \bar{A} \\
\mathcal{B}^{-}(A) & =\mathcal{B}(A) \cap A \\
\mathcal{B}(A) & =\mathcal{C}(A) \cap \mathcal{C}(\bar{A})
\end{aligned}
$$

\section{Quasi-Discrete Closure Spaces}

In this section we see how a closure space may be derived starting from a binary relation, that is, a graph. The following comes from [17].

Definition 4. Consider a set $X$ and a relation $R \subseteq X \times X$. A closure operator is obtained from $R$ as $\mathcal{C}_{R}(A)=A \cup\{x \in X \mid \exists a \in A$. $(a, x) \in R\}$. 
Remark 1. One could also change Definition 4 so that $\mathcal{C}_{R}(A)=A \cup\{x \in X \mid$ $\exists a \in A .(x, a) \in R\}$, which actually is the definition of [17]. This does not affect the theory presented in the paper. Indeed, one obtains the same results by replacing $R$ with $R^{-1}$ in statements of theorems that explicitly use $R$, and are not invariant under such change. By our choice, closure represents the "least possible enlargement" of a set of nodes.

Proposition 2. The pair $\left(X, \mathcal{C}_{R}\right)$ is a closure space.

Closure operators obtained by Definition 4 are not necessarily idempotent. Lemma 11 in [17] provides a necessary and sufficient condition, that we rephrase below. We let $R^{=}$denote the reflexive closure of $R$ (that is, the least relation that includes $R$ and is reflexive).

Lemma 2. $\mathcal{C}_{R}$ is idempotent if and only if $R^{=}$is transitive.

Note that, when $R$ is transitive, so is $R^{=}$, thus $\mathcal{C}_{R}$ is idempotent. The viceversa is not true, e.g., when $(x, y) \in R,(y, x) \in R$, but $(x, x) \notin R$.

Remark 2. In topology, open sets play a fundamental role. However, the situation is different in closure spaces derived from a relation $R$. For example, in the case of a closure space derived from a connected symmetric relation, the only open sets are the whole space, and the empty set.

Proposition 3. Given $R \subseteq X \times X$, in the space $\left(X, \mathcal{C}_{R}\right)$, we have:

$$
\begin{aligned}
\mathcal{I}(A) & =\{x \in A \mid \neg \exists a \in \bar{A} .(a, x) \in R\} \\
\mathcal{B}^{-}(A) & =\{x \in A \mid \exists a \in \bar{A} .(a, x) \in R\} \\
\mathcal{B}^{+}(A) & =\{x \in \bar{A} \mid \exists a \in A .(a, x) \in R\}
\end{aligned}
$$

We note in passing that [16] provides an alternative definition of boundaries for closure spaces obtained from Definition 4, and proves that it coincides with the topological definition (our Definition 3). Closure spaces derived from a relation can be characterised as quasi-discrete spaces (see also Lemma 9 of [17] and the subsequent statements).

Definition 5. A closure space is quasi-discrete if and only if one of the following equivalent conditions holds: i) each $x \in X$ has a minimal neighbourhood ${ }^{3}$ $N_{x}$; ii) for each $A \subseteq X, \mathcal{C}(A)=\bigcup_{a \in A} \mathcal{C}(\{a\})$.

Theorem 1. (Theorem 1 in [17]) A closure space $(X, \mathcal{C})$ is quasi-discrete if and only if there is a relation $R \subseteq X \times X$ such that $\mathcal{C}=\mathcal{C}_{R}$.

\footnotetext{
${ }^{3}$ A minimal neighbourhood of $x$ is a set that is a neighbourhood of $x$ (Definition 2 (2)) and is included in all other neighbourhoods of $x$.
} 


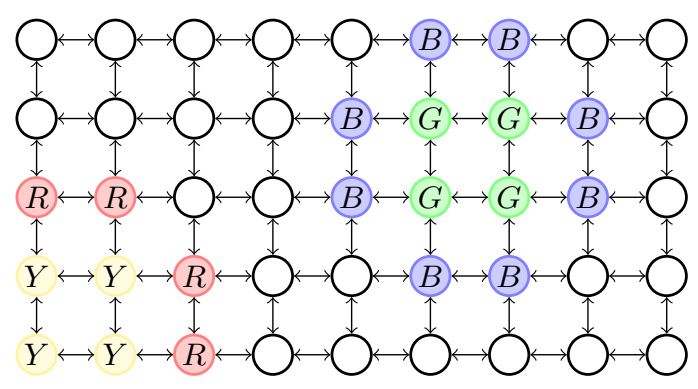

Fig. 1. A graph inducing a quasi-discrete closure space

Example 1. Every graph induces a quasi-discrete closure space. For instance, we can consider the (undirected) graph depicted in Figure 1. Let $R$ be the (symmetric) binary relation induced by the graph edges, and let $Y$ and $G$ denote the set of yellow and green nodes, respectively. The closure $\mathcal{C}_{R}(Y)$ consists of all yellow and red nodes, while the closure $\mathcal{C}_{R}(G)$ contains all green and blue nodes. The interior $\mathcal{I}(Y)$ of $Y$ contains a single node, i.e. the one located at the bottom-left in Figure 1. On the contrary, the interior $\mathcal{I}(G)$ of $G$ is empty. Indeed, we have that $\mathcal{B}(G)=\mathcal{C}(G)$, while $\mathcal{B}^{-}(G)=G$ and $\mathcal{B}^{+}(G)$ consists of the blue nodes.

\section{A Spatial Logic for Closure Spaces}

In this section we present a spatial logic that can be used to express properties of closure spaces. The logic features two spatial operators: a "one step" modality, turning closure into a logical operator, and a binary until operator, which is interpreted spatially. Before introducing the complete framework, we first discuss the design of an until operator $\phi \mathcal{U} \psi$.

The spatial logical operator $\mathcal{U}$ is interpreted on points of a closure space. The basic idea is that point $x$ satisfies $\phi \mathcal{U} \psi$ whenever it is included in an area $A$ satisfying $\phi$, and there is "no way out" from $A$ unless passing through an area $B$ that satisfies $\psi$. For instance, if we consider the model of Figure 1, yellow nodes satisfy yellow $\mathcal{U}$ red while green nodes satisfy green $\mathcal{U}$ blue. To turn this intuition into a mathematical definition, one should clarify the meaning of the words area, included, passing, in the context of closure spaces.

In order to formally define our logic, and the until operator in particular, we first need to introduce the notion of model, providing a context of evaluation for the satisfaction relation, as in $\mathcal{M}, x \models \phi \mathcal{U} \psi$. From now on, fix a (finite or countable) set $P$ of proposition letters.

Definition 6. A closure model is a pair $\mathcal{M}=((X, \mathcal{C}), \mathcal{V})$ consisting of a closure space $(X, \mathcal{C})$ and a valuation $\mathcal{V}: P \rightarrow 2^{X}$, assigning to each proposition letter the set of points where the proposition holds. 
When $(X, \mathcal{C})$ is a topological space (that is, $\mathcal{C}$ is idempotent), we call $\mathcal{M}$ a topological model, in line with [24], and [1], where the topological until operator is presented. We recall it below.

Definition 7. The topological until operator $\mathcal{U}_{T}$ is interpreted in a topological model $\mathcal{M}$ as $\mathcal{M}, x \models \phi \mathcal{U}_{T} \psi \Longleftrightarrow \exists A$ open $. x \in A \wedge \forall y \in A . \mathcal{M}, y=\phi \wedge \forall z \in$ $\mathcal{B}(A) . \mathcal{M}, z \models \psi$.

The intuition behind this definition is that one seeks for an area $A$ (which, topologically speaking, could sensibly be an open set) where $\phi$ holds, and that is completely surrounded by points where $\psi$ holds. Unfortunately, Definition 7 cannot be translated directly to closure spaces, even if all the used topological notions have a counterpart in the more general setting of closure spaces. Open sets in closure spaces are often too coarse (see Remark 2). For this reason, we can modify Definition 7 by not requiring $A$ to be an open set. However, the usage of $\mathcal{B}$ in Definition 7 is not satisfactory either. By Proposition 1 we have $\mathcal{B}(A)=\mathcal{B}^{+}(A) \cup \mathcal{B}^{-}(A)$, where $\mathcal{B}^{-}(A)$ is included in $A$ while $\mathcal{B}^{+}(A)$ is in $\bar{A}$. For instance, when $\mathcal{B}$ is used in Definition 7 , we have that the green nodes in Figure 1 do not satisfy green $\mathcal{U}_{T}$ blue. Indeed, as we remarked in Example 1, the boundary of the set $G$ of green nodes coincide with the closure of $G$ that contains both green and blue nodes.

A more satisfactory definition can be obtained by letting $\mathcal{B}^{+}$play the same role as $\mathcal{B}$ in Definition 7 and not requiring $A$ to be an open set. We shall in fact require that $\phi$ is satisfied by all the points of $A$, and that in $\mathcal{B}^{+}(A), \psi$ holds. This allows us to ensure that there are no "gaps" between the region satisfying $\phi$ and that satisfying $\psi$.

\subsection{Syntax and Semantics of $S L C S$}

We can now define SLCS: a Spatial Logic for Closure Spaces. The logic features boolean operators, a "one step" modality, turning closure into a logical operator, and a spatially interpreted until operator. More precisely, as we shall see, the $S L C S$ formula $\phi \mathcal{U} \psi$ requires $\phi$ to hold at least on one point. The operator is similar to a weak until in temporal logics terminology, as there may be no point satisfying $\psi$, if $\phi$ holds everywhere.

Definition 8. The syntax of SLCS is defined by the following grammar, where $p$ ranges over $P$ :

$$
\Phi::=p|\top| \neg \Phi|\Phi \wedge \Phi| \diamond \Phi \mid \Phi \mathcal{U} \Phi
$$

Here, $\top$ denotes true, $\neg$ is negation, $\wedge$ is conjunction, $\diamond$ is the closure operator, and $\mathcal{U}$ is the until operator. Closure (and interior, see Figure 2) operators come from the tradition of topological spatial logics [24]. 


$$
\begin{array}{lll}
\perp \quad \triangleq \neg \top & \phi \vee \psi \triangleq \neg(\neg \phi \wedge \neg \psi) & \square \phi \triangleq \neg(\diamond \neg \phi) \\
\partial \phi \triangleq(\triangleright \phi) \wedge(\neg \square \phi) & \partial^{-} \phi \triangleq \phi \wedge(\neg \square \phi) & \partial^{+} \phi \triangleq(\diamond \phi) \wedge(\neg \phi) \\
\phi \mathcal{R} \psi \triangleq \neg((\neg \psi) \mathcal{U}(\neg \phi)) & \mathcal{G} \phi \triangleq \phi \mathcal{U} \perp & \mathcal{F} \phi \triangleq \neg \mathcal{G}(\neg \phi)
\end{array}
$$

Fig. 2. $S L C S$ derivable operators

Definition 9. Satisfaction $\mathcal{M}, x \models \phi$ of formula $\phi$ at point $x$ in model $\mathcal{M}=$ $((X, \mathcal{C}), \mathcal{V})$ is defined, by induction on terms, as follows:

$$
\begin{array}{ll}
\mathcal{M}, x=p & \Longleftrightarrow x \in \mathcal{V}(p) \\
\mathcal{M}, x=\top \quad \Longleftrightarrow \text { true } \\
\mathcal{M}, x=\neg \phi & \Longleftrightarrow \mathcal{M}, x \mid \models \phi \\
\mathcal{M}, x=\phi \wedge \psi & \Longleftrightarrow \mathcal{M}, x \models \phi \text { and } \mathcal{M}, x \models \psi \\
\mathcal{M}, x=\nabla \phi & \Longleftrightarrow x \in \mathcal{C}(\{y \in X \mid \mathcal{M}, y \models \phi\}) \\
\mathcal{M}, x=\phi \mathcal{U} \psi & \Longleftrightarrow \exists A \subseteq X . x \in A \wedge \forall y \in A . \mathcal{M}, y \models \phi \wedge \\
&
\end{array}
$$

In Figure 2, we present some derived operators. Besides standard logical connectives, the logic can express the interior $(\square \phi)$, the boundary $(\partial \phi)$, the interior boundary $\left(\partial^{-} \phi\right)$ and the closure boundary $\left(\partial^{+} \phi\right)$ of the set of points satisfying formula $\phi$. Moreover, by appropriately using the until operator, operators concerning reachability $(\phi \mathcal{R} \psi)$, global satisfaction $(\mathcal{G} \phi)$ and possible satisfaction $(\mathcal{F} \phi)$ can be derived.

To clarify the expressive power of $\mathcal{U}$ and operators derived from it we provide Theorem 2 and Theorem 3, giving a formal meaning to the idea of "way out" of $\phi$, and providing an interpretation of $\mathcal{U}$ in terms of paths.

Definition 10. A closure-continuous function $f:\left(X_{1}, \mathcal{C}_{1}\right) \rightarrow\left(X_{2}, \mathcal{C}_{2}\right)$ is a function $f: X_{1} \rightarrow X_{2}$ such that, for all $A \subseteq X_{1}, f\left(\mathcal{C}_{1}(A)\right) \subseteq \mathcal{C}_{2}(f(A))$.

Definition 11. Consider a closure space $(X, \mathcal{C})$, and the quasi-discrete space $\left(\mathbb{N}, \mathcal{C}_{\text {Succ }}\right)$, where $(n, m) \in$ Succ $\Longleftrightarrow m=n+1$. A (countable) path in $(X, \mathcal{C})$ is a closure-continuous function $p:\left(\mathbb{N}, \mathcal{C}_{\text {Succ }}\right) \rightarrow(X, \mathcal{C})$. We call $p$ a path from $x$, and write $p: x \rightsquigarrow \infty$, when $p(0)=x$. We write $y \in p$ whenever there is $l \in \mathbb{N}$ such that $p(l)=y$. We write $p: x \underset{y}{\stackrel{A}{\leftrightarrow}} \infty$ when $p$ is a path from $x$, and there is $l$ with $p(l)=y$ and for all $l^{\prime} \leq l . p\left(l^{\prime}\right) \in A$.

Theorem 2. If $\mathcal{M}, x \models \phi \mathcal{U} \psi$, then for each $p: x \rightsquigarrow \infty$ and $l$, if $\mathcal{M}, p(l) \models \neg \phi$, there is $k \in\{1, \ldots, l\}$ such that $\mathcal{M}, p(k) \models \psi$.

Theorem 2 can be strengthened to a necessary and sufficient condition in the case of models based on quasi-discrete spaces. First, we establish that paths in a quasi-discrete space are also paths in its underlying graph.

Lemma 3. Given path $p$ in a quasi-discrete space $\left(X, \mathcal{C}_{R}\right)$, for all $i \in \mathbb{N}$ with $p(i) \neq p(i+1)$, we have $(p(i), p(i+1)) \in R$, i.e., the image of $p$ is a (graph theoretical, infinite) path in the graph of $R$. Conversely, each path in the graph of $R$ uniquely determines a path in the sense of Definition 11. 


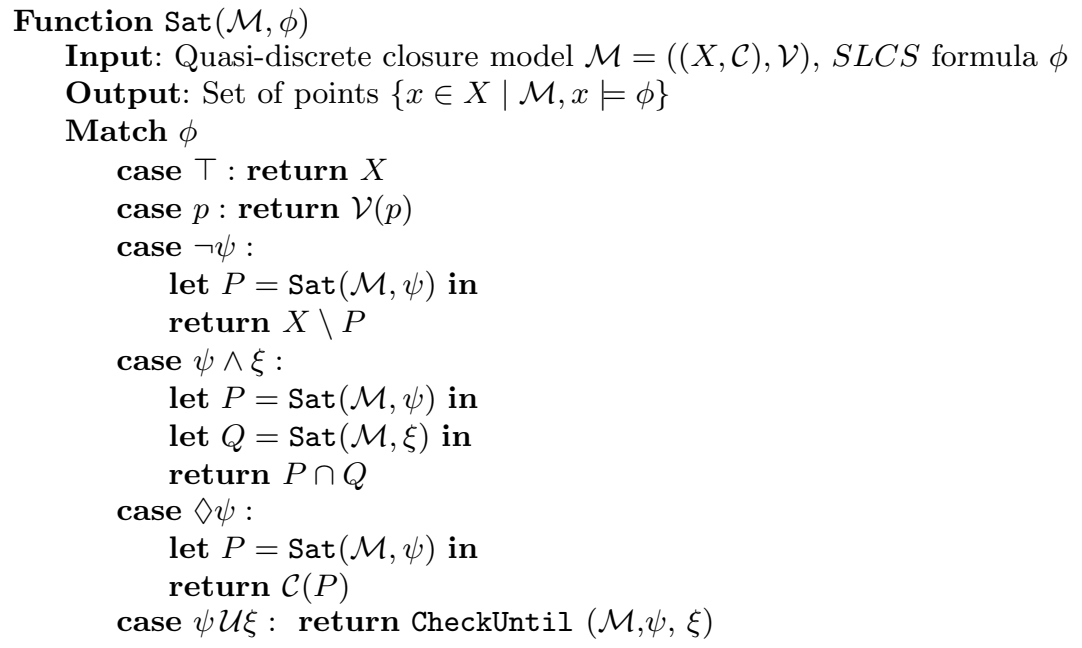

Algorithm 1. Decision procedure for the model checking problem

Theorem 3. In a quasi-discrete closure model $\mathcal{M}, \mathcal{M}, x \models \phi \mathcal{U} \psi$ if and only if $\mathcal{M}, x \models \phi$, and for each path $p: x \rightsquigarrow \infty$ and $l \in \mathbb{N}$, if $\mathcal{M}, p(l) \models \neg \phi$, there is $k \in\{1, \ldots, l\}$ such that $\mathcal{M}, p(k) \models \psi$.

Remark 3. Directly from Theorem 3 and from the definitions in Figure 2 we have also that in a quasi-discrete closure model $\mathcal{M}$ :

1. $\mathcal{M}, x \models \phi \mathcal{R} \psi$ iff. there is $p: x \rightsquigarrow \infty$ and $k \in \mathbb{N}$ such that $\mathcal{M}, p(k) \models \psi$ and for each $j \in\{1, \ldots, k\} \mathcal{M}, p(j) \models \phi$;

2. $\mathcal{M}, x \models \mathcal{G} \phi$ iff. for each $p: x \rightsquigarrow \infty$ and $i \in \mathbb{N}, \mathcal{M}, p(i) \models \phi$;

3. $\mathcal{M}, x \models \mathcal{F} \phi$ iff. there is $p: x \rightsquigarrow \infty$ and $i \in \mathbb{N}$ such that $\mathcal{M}, p(i) \models \phi$.

Note that, a point $x$ satisfies $\phi \mathcal{R} \psi$ if and only if either $\psi$ is satisfied by $x$ or there exists a sequence of points after $x$, all satisfying $\phi$, leading to a point satisfying both $\psi$ and $\phi$. In the second case, it is not required that $x$ satisfies $\phi$.

\section{$5 \quad$ Model Checking SLCS Formulas}

In this section we present a model checking algorithm for $S L C S$, which is a variant of standard CTL model checking [3]. Function sat, presented in Algorithm 1, takes as input a finite quasi-discrete model $\mathcal{M}=\left(\left(X, \mathcal{C}_{R}\right), \mathcal{V}\right)$ and an $S L C S$ formula $\phi$, and returns the set of all points in $X$ satisfying $\phi$. The function is inductively defined on the structure of $\phi$ and, following a bottom-up approach, computes the resulting set via an appropriate combination of the recursive invocations of Sat on the sub-formulas of $\phi$. When $\phi$ is $\top, p, \neg \psi$ or $\psi \wedge \xi$, 


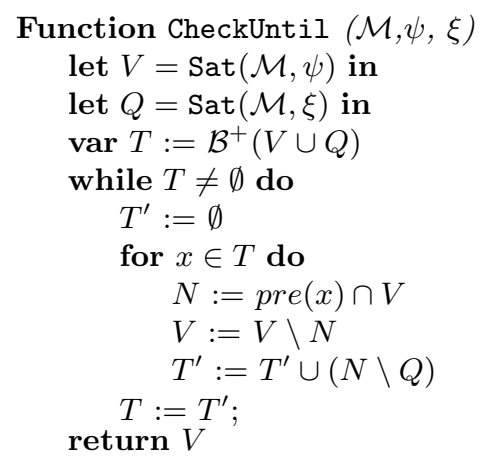

Algorithm 2. Checking until formulas in a quasi-discrete closure space

definition of $\operatorname{Sat}(\mathcal{M}, \phi)$ is as expected. To compute the set of points satisfying $\checkmark \psi$, the closure operator $\mathcal{C}$ of the space is applied to the set of points satisfying $\psi$.

When $\phi$ is of the form $\psi \mathcal{U} \xi$, function Sat relies on the function CheckUntil defined in Algorithm 2. This function takes as parameters a model $\mathcal{M}$ and two $S L C S$ formulas $\psi$ and $\xi$ and computes the set of points in $\mathcal{M}$ satisfying $\psi \mathcal{U} \xi$ by removing from $V=\operatorname{Sat}(\mathcal{M}, \psi)$ all the bad points. A point is bad if there exists a path passing through it, that leads to a point satisfying $\neg \psi$ without passing through a point satisfying $\xi$. Let $Q=\operatorname{Sat}(\mathcal{M}, \xi)$ be the set of points in $\mathcal{M}$ satisfying $\xi$. To identify the bad points in $V$ the function CheckUntil performs a backward search from $T=\mathcal{B}^{+}(V \cup Q)$. Note that any path exiting from $V \cup Q$ has to pass through points in $T$. Moreover, the latter only contains points that satisfy neither $\psi$ nor $\xi$. Until $T$ is empty, function CheckUntil first picks an element $x$ in $T$ and then removes from $V$ the set of (bad) points $N$ that can reach $x$ in one step. To compute the set $N$ we use the function $\operatorname{pre}(x)=\{y \in X \mid(y, x) \in R\}{ }^{4}$ At the end of each iteration the set $T$ is updated by considering the set of new discovered bad points.

Lemma 4. Let $X$ a finite set and $R \subseteq X \times X$. For any finite quasi-discrete model $\mathcal{M}=\left(\left(X, \mathcal{C}_{R}\right), \mathcal{V}\right)$ and $S L C S$ formula $\phi$ with $k$ operators, Sat terminates in $\mathcal{O}(k \cdot(|X|+|R|))$ steps.

Theorem 4. For any finite quasi-discrete closure model $\mathcal{M}=((X, \mathcal{C}), \mathcal{V})$ and $S L C S$ formula $\phi, x \in \operatorname{Sat}(\mathcal{M}, \phi)$ if and only if $\mathcal{M}, x \models \phi$.

\section{A Model Checker for Spatial Logics}

The algorithm described in Section 5 is available as a proof-of-concept tool ${ }^{5}$. The tool, implemented using the functional language OCaml, contains a generic

\footnotetext{
${ }^{4}$ Function pre can be pre-computed when the relation $R$ is loaded from the input.

${ }^{5}$ Web site: http://www.github.com/vincenzoml/slcs.
} 


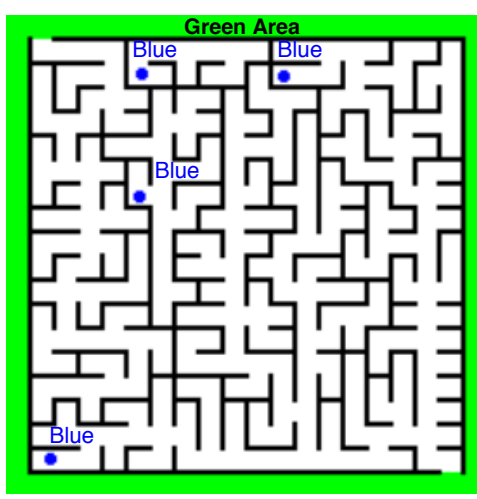

Fig. 3. A maze

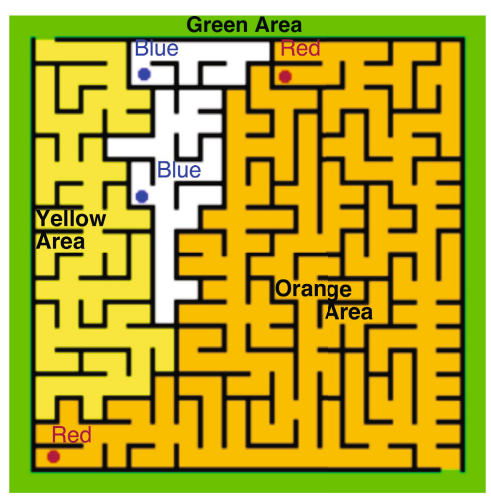

Fig. 4. Model checker output

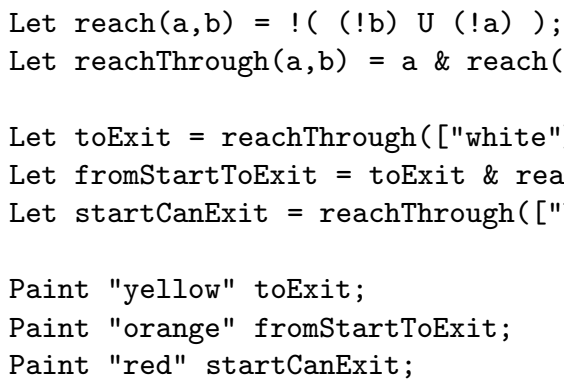

Fig. 5. Input to the model checker

implementation of a global model-checker using closure spaces, parametrised by the type of models.

An example of the tool usage is to approximately identify regions of interest on a digital picture (e.g., a map, or a medical image), using spatial formulas. In this case, digital pictures are treated as quasi-discrete models in the plane $\mathbb{Z} \times \mathbb{Z}$. The language of propositions is extended to simple formulas dealing with colour ranges, in order to cope with images where there are different shades of certain colours.

In Figure 3 we show a digital picture of a maze. The green area is the exit. The blue areas are start points. The input of the tool is shown in Figure 5, where the Paint command is used to invoke the global model checker and colour points satisfying a given formula. Three formulas, making use of the until operator, are used to identify interesting areas. The output of the tool is in Figure 4. The colour red denotes start points from which the exit can be reached. Orange and yellow indicate the two regions through which the exit can be reached, including and excluding a start point, respectively. 


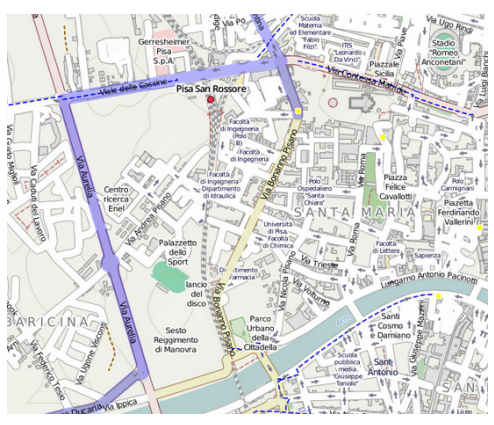

Fig. 6. Input: the map of a town

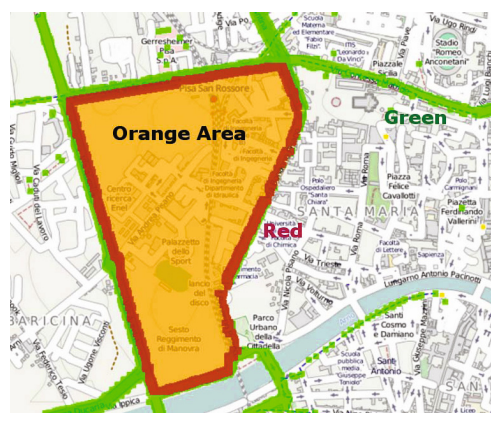

Fig. 7. Output of the tool

In Figure 6 we show a digital image ${ }^{6}$ depicting a portion of the map of Pisa, featuring a red circle which denotes a train station. Streets of different importance are painted with different colors in the map. The model checker is used to identify the area surrounding the station which is delimited by main streets, and the delimiting main streets. The output of the tool is shown in Figure 7, where the station area is coloured in orange, the surrounding main streets are red, and other main streets are in green. We omit the source code of the model checking session for space reasons (see the source code of the tool). As a mere hint on how practical it is to use a model checker for image analysis, the execution time on our test image, consisting of about 250000 pixels, is in the order of ten seconds on a standard laptop equipped with a $2 \mathrm{Ghz}$ processor.

\section{Conclusions and Future Work}

In this paper, we have presented a methodology to verify properties that depend upon space. We have defined an appropriate logic, stemming from the tradition of topological interpretations of modal logics, dating back to earlier logicians such as Tarski, where modalities describe neighbourhood. The topological definitions have been lifted to a more general setting, also encompassing discrete, graphbased structures. The proposed framework has been extended with a spatial variant of the until operator, and we have also defined an efficient model checking procedure, which is implemented in a proof-of-concept tool.

As future work, we first of all plan to merge the results presented in this paper with temporal reasoning. This integration can be done in more than one way. It is not difficult to consider "snapshot" models consisting of a temporal model (e.g., a Kripke frame) where each state is in turn a closure model, and atomic formulas of the temporal fragment are replaced by spatial formulas. The various possible combinations of temporal and spatial operators, in linear and branching time, are examined for the case of topological models and basic modal

\footnotetext{
${ }^{6}$ (c) OpenStreetMap contributors - http://www.openstreetmap.org/copyright.
} 
formulas in [18]. Snapshot models may be susceptible to state-space explosion problems as spatial formulas could need to be recomputed at every state. On the other hand, one might be able to exploit the fact that changes of space over time are incremental and local in nature. Promising ideas are presented both in [17], where principles of "continuous change" are proposed in the setting of closure spaces, and in [20] where spatio-temporal models are generated by locallyscoped update functions, in order to describe dynamic systems. In the setting of collective adaptive systems, it will be certainly needed to extend the basic framework we presented with metric aspects (e.g., distance-bounded variants of the until operator), and probabilistic aspects, using atomic formulas that are probability distributions. A thorough investigation of these issues will be the object of future research.

A challenge in spatial and spatio-temporal reasoning is posed by recursive spatial formulas, a la $\mu$-calculus, especially on infinite structures with relatively straightforward generating functions (think of fractals, or fluid flow analysis of continuous structures). Such infinite structures could be described by topologically enhanced variants of $\omega$-automata. Classes of automata exist living in specific topological structures; an example is given by nominal automata (see e.g., $[4,14,21])$, that can be defined using presheaf toposes [13]. This standpoint could be enhanced with notions of neighbourhood coming from closure spaces, with the aim of developing a unifying theory of languages and automata describing space, graphs, and process calculi with resources.

\section{References}

1. Aiello, M.: Spatial Reasoning: Theory and Practice. PhD thesis, Institute of Logic, Language and Computation, University of Amsterdam (2002)

2. Aiello, M., Pratt-Hartmann, I., van Benthem, J. (eds.): Handbook of Spatial Logics. Springer (2007)

3. Baier, C., Katoen, J.P.: Principles of model checking. MIT Press (2008)

4. Bojanczyk, M., Klin, B., Lasota, S.: Automata with group actions. In: LICS, pp. 355-364. IEEE Computer Society Press (2011)

5. Bortolussi, L., Hillston, J., Latella, D., Massink, M.: Continuous approximation of collective system behaviour: A tutorial. Perform. Eval. 70(5), 317-349 (2013)

6. Caires, L., Cardelli, L.: A spatial logic for concurrency (part I). Information and Computation 186(2), 194-235 (2003)

7. Cardelli, L., Gardner, P., Ghelli, G.: A spatial logic for querying graphs. In: Widmayer, P., Triguero, F., Morales, R., Hennessy, M., Eidenbenz, S., Conejo, R. (eds.) ICALP 2002. LNCS, vol. 2380, pp. 597-610. Springer, Heidelberg (2002)

8. Cardelli, L., Gordon, A.D.: Anytime, anywhere: Modal logics for mobile ambients. In: POPL, pp. 365-377. ACM (2000)

9. Chaintreau, A., Le Boudec, J., Ristanovic, N.: The age of gossip: Spatial mean field regime. In: SIGMETRICS, pp. 109-120. ACM, New York (2009)

10. Ciancia, V., Latella, D., Loreti, M., Massink, M.: Specifying and verifying properties of space - extended version. CoRR, abs/1406.6393 (2014)

11. De Nicola, R., Ferrari, G.L., Pugliese, R.: Klaim: A kernel language for agents interaction and mobility. IEEE Trans. Software Eng. 24(5), 315-330 (1998) 
12. Del Bimbo, A., Vicario, E., Zingoni, D.: Symbolic description and visual querying of image sequences using spatio-temporal logic. IEEE Trans. Knowl. Data Eng. 7(4), 609-622 (1995)

13. Fiore, M.P., Staton, S.: Comparing operational models of name-passing process calculi. Information and Computation 204(4), 524-560 (2006)

14. Gabbay, M.J., Ciancia, V.: Freshness and name-restriction in sets of traces with names. In: Hofmann, M. (ed.) FOSSACS 2011. LNCS, vol. 6604, pp. 365-380. Springer, Heidelberg (2011)

15. Gadducci, F., Lluch Lafuente, A.: Graphical encoding of a spatial logic for the $\pi$-calculus. In: Mossakowski, T., Montanari, U., Haveraaen, M. (eds.) CALCO 2007. LNCS, vol. 4624, pp. 209-225. Springer, Heidelberg (2007)

16. Galton, A.: The mereotopology of discrete space. In: Freksa, C., Mark, D.M. (eds.) COSIT 1999. LNCS, vol. 1661, pp. 251-266. Springer, Heidelberg (1999)

17. Galton, A.: A generalized topological view of motion in discrete space. Theoretical Computer Science 305(1-3), 111 (2003)

18. Kontchakov, R., Kurucz, A., Wolter, F., Zakharyaschev, M.: Spatial logic + temporal $\operatorname{logic}=$ ? In: Aiello, et al. (eds.) [2], pp. 497-564

19. Kovalevsky, V.A.: Geometry of Locally Finite Spaces: Computer Agreeable Topology and Algorithms for Computer Imagery. House Dr. Baerbel Kovalevski (2008)

20. Kremer, P., Mints, G.: Dynamic topological logic. In: Aiello, et al. (eds.) [2], pp. 565-606

21. Kurz, A., Suzuki, T., Tuosto, E.: On nominal regular languages with binders. In: Birkedal, L. (ed.) FOSSACS 2012. LNCS, vol. 7213, pp. 255-269. Springer, Heidelberg (2012)

22. Rosenfeld, A.: Digital topology. The American Mathematical Monthly 86(8), 621-630 (1979)

23. Smyth, M.B., Webster, J.: Discrete spatial models. In: Aiello, et al. (eds.) [2], pp. $713-798$

24. van Benthem, J., Bezhanishvili, G.: Modal logics of space. In: Handbook of Spatial Logics, pp. 217-298 (2007)

25. Yung Kong, T., Rosenfeld, A.: Digital topology: Introduction and survey. Computer Vision, Graphics, and Image Processing 48(3), 357-393 (1989) 